\title{
An Optimal Stopping Problem for Jump Diffusion Logistic Population Model
}

\author{
Yang Sun ${ }^{1}$ and Xiaohui $\mathrm{Ai}^{2}$ \\ ${ }^{1}$ School of Applied Science, Harbin University of Science and Technology, Harbin 150080, China \\ ${ }^{2}$ Department of Mathematics, Northeast Forestry University, Harbin 150040, China \\ Correspondence should be addressed to Yang Sun; sunysy@126.com
}

Received 11 May 2016; Revised 13 July 2016; Accepted 21 July 2016

Academic Editor: Khaled Bahlali

Copyright (c) 2016 Y. Sun and X. Ai. This is an open access article distributed under the Creative Commons Attribution License, which permits unrestricted use, distribution, and reproduction in any medium, provided the original work is properly cited.

\begin{abstract}
This paper examines an optimal stopping problem for the stochastic (Wiener-Poisson) jump diffusion logistic population model. We present an explicit solution to an optimal stopping problem of the stochastic (Wiener-Poisson) jump diffusion logistic population model by applying the smooth pasting technique (Dayanik and Karatzas, 2003; Dixit, 1993). We formulate this as an optimal stopping problem of maximizing the expected reward. We express the critical state of the optimal stopping region and the optimal value function explicitly.
\end{abstract}

\section{Introduction}

The theory of optimal stopping is widely applied in many fields such as finance, insurance, and bioeconomics. Optimal stopping problems for lots of models have been put forward to meet the actual need. Bioeconomic resource models incorporating random fluctuations in either population size or model parameters have been the subject of much interest. The optimal stopping problem is very important in mathematical bioeconomics and has been extensively studied;see Clark [1], Dayanik and Karatzas [2], Dai and Kwok [3], Presman and Sonin [4], Christensen and Irle [5], and so forth. A very classic and successful model for population growth in mathematics is logistic model

$$
d X_{t}=\left(r X_{t}-b X_{t}^{2}\right) d t
$$

where $X_{t}$ denotes the density of resource population at time $t, r>0$ is called the intrinsic growth rate, and $b=r / K>$ 0 ( $K$ is the environmental carrying capacity). The logistic model is used widely to real data; however, it is too simple to provide a better simulation of the real world since there are some uncertainties, such as environment and financial effect, modeled by Gaussian white noise. Hence, the stochastic logistic differential equation is introduced to handle these problems; that is,

$$
\begin{aligned}
& d X_{t}=\left(r X_{t}-b X_{t}^{2}\right) d t+\mu X_{t} d B_{t}, \\
& X_{0}=x \geq 0, t \geq 0,
\end{aligned}
$$

where the constants $r, b$ are mentioned in (1), $\mu$ is a measure of the size of the noise in the system, and $B_{t}$ is 1-dimensional Brownian motion defined on a complete probability space $\left(\Omega, \mathfrak{F},\left\{\mathfrak{F}_{t}\right\}_{t>0}, \mathbb{P}\right)$ satisfing the usual conditions. There are so many extensive researches in literature, such as Lungu and Øksendal [6], Sun and Wang [7], Liu and Wang [8], and Liu and Wang $[9,10]$.

Furthermore, large and sudden fluctuations in environmental fluctuations can not modeled by the Gaussian white noise, for examples, hurricanes, disasters, and crashes. A Poisson jump stochastic equation can explain the sudden changes. In this paper, we will concentrate on the stochastic logistic population model with Poisson jump

$$
\begin{aligned}
d X_{t}= & \left(r X_{t}-b X_{t}^{2}\right) d t+\mu X_{t} d B_{t} \\
& +\int_{0}^{\infty} c X_{t^{-}} \widetilde{N}(d t, d z), \quad X_{0}=x \geq 0, t \geq 0,
\end{aligned}
$$


where $X\left(t^{-}\right)$is the left limit of $X(t), r, b, \mu$, and $B_{t}$ are defined in (2), $c$ is a bounded constant, $N$ is a Poisson counting measure with characteristic measure $v$ on a measurable subset $Y$ of $(0, \infty)$ with $v(Y)<\infty$, and $\widetilde{N}(d t, d z)=N(d t, d z)-$ $v(d z) d t$. Throughout the paper, we assume that $B$ and $N$ are independent. More discussions of the stochastic jump diffusion model are given by Ryan and Hanson [11], Wee [12], Kunita [13], and Bao et al. [14] and the references therein.

Many methods, such as Fokker-Planck equations, time averaging methods, and stochastic calculus are used on optimal harvesting problems for model (2); all the aforementioned works can be found in Alvarez and Shepp [15], Li and Wang [16], and Li et al. [17]. To my best knowledge, even for model (2), there is little try by using optimal stopping theory on optimal harvesting problems; therefore, in this paper, we will try the optimal stopping approach to solve the optimal harvesting problem for model (3), which is the motivation of the paper.

The paper is organized as follows. In Section 2, in order to find the optimal value function and the optimal stopping region, we formulate the problem and suppose we have a fish factory with a population (e.g., a fish population in a pond) whose size $X_{t}$ at time $t$ is described by the stochastic jump diffusion model (3), as a stopping problem. In Section 3, an explicit function for the value function is verified; meanwhile, the optimal stopping time and the optimal stopping region are expressed.

\section{Description of Problem}

Suppose the population with size $X_{t}$ at time $t$ is given by the stochastic logistic population model with Poisson jump

$$
\begin{aligned}
d X_{t}= & \left(r X_{t}-b X_{t}^{2}\right) d t+\mu X_{t} d B_{t} \\
& +\int_{0}^{\infty} c X_{t^{-}} \widetilde{N}(d t, d z)
\end{aligned}
$$

It can be proved that if $r>0, b>0$ and $\mu, c$ are bounded constants, then (4) has a unique positive solution $X_{t}$ defined by

$$
X_{t}^{x}=\frac{\Phi(t)}{1 / x+b \int_{0}^{t} \Phi(s) d s}
$$

where

$$
\begin{gathered}
\Phi(t)=\exp \left(\left(r-\frac{1}{2} \mu^{2}\right) t+\mu B_{t}\right. \\
+\int_{Y}(\ln (1+c)-c) t v(d z) \\
\left.+\int_{0}^{t} \int_{Y} \ln (1+c) \widetilde{N}(d s, d z)\right)
\end{gathered}
$$

for all $t \geq 0$ (see Bao et al. [14]) and note that $0 \leq X_{t}<K$.

Supposing that the population is, say, a fish population in a pond, the goal of this paper, the optimal strategy for selling a fish factory, can be considered as an optimal stopping problem: find $v^{*}(s, x)$ and $\tau^{*}$ such that

$$
\begin{aligned}
v^{*}(s, x) & =\sup _{\tau} E^{x}\left[e^{-\rho \tau}\left(p X_{\tau}^{\beta}-q\right)\right] \\
& =E^{x}\left[e^{-\rho \tau^{*}}\left(p X_{\tau^{*}}^{\beta}-q\right)\right], \quad \beta, p, q>0 ;
\end{aligned}
$$

the sup is taken over all stopping times $\tau$ of the process $X_{t}$, $t>0$ with the reward function

$$
R(s, x)=e^{-\rho s}\left(p x^{\beta}-q\right)
$$

where the discounted exponent is $\rho>0, e^{-\rho s}\left(p x^{\beta}-q\right)$ is the profit of selling fish at time $\tau$, and $q$ represents a fixed fee and it is nature to assume that $q<K$. $E^{x}$ denotes the expectation with respect to the probability law $P^{x}$ of the process $X_{t}, t \geq 0$ starting at $X_{0}=x>0$.

We will search for an optimal stopping time $\tau^{*}$ given in (30) with the optimal stopping boundary $x^{*}$ from (23) on the interval $(0, K)$ such that we can obtain the optimal profit $v^{*}$ in (28) and the optimal stopping region $A$ in (29). Note that it is trivial that the initial value $x \leq q$, so we further assume $x>q$.

\section{Analysis}

For the jump diffusion logistic population model

$$
\begin{aligned}
d X_{t}= & \left(r X_{t}-b X_{t}^{2}\right) d t+\mu X_{t} d B_{t} \\
& +\int_{0}^{\infty} c X_{t^{-}} \widetilde{N}(d t, d z),
\end{aligned}
$$

and applying the Itô formula to a $C^{2}$-function $f$ such that $\mathbb{E}\left[\int_{0}^{t} \int_{Y}|f(t, z)| v(d z) d t\right]<\infty$ and $f^{\prime}, f^{\prime \prime}$ are bounded, we have the infinitesimal generator of the process $f\left(X_{t}\right)$, that is

$$
\begin{aligned}
& {[\mathscr{L} f](x)} \\
& =\left(r x-b x^{2}\right) f^{\prime}(x)+\frac{1}{2} \mu^{2} x^{2} f^{\prime \prime}(x) \\
& \quad+\int_{Y}\left\{f(x+c x)-f(x)-c x f^{\prime}(x)\right\} v(d z) ;
\end{aligned}
$$

provided that $\int_{Y}\left\{f(x+c x)-f(x)-c x f^{\prime}(x)\right\} v(d z)$ is well defined since

$$
\left|f(x+c x)-f(x)-c x f^{\prime}(x)\right| \leq \frac{1}{2} c^{2} x^{2}\left|f^{\prime \prime}(x)\right|
$$

and $c x$ are bounded.

Now let us consider a function equation

$$
[\mathscr{L} f](x)=0, \quad x \in \mathbb{R}_{+} .
$$

We can try a solution of the form $f(x)=\alpha x^{\beta}, x \in \mathbb{R}_{+}$to determine the unknown function; that is

$$
\begin{aligned}
& {[\mathscr{L} f](x)=\alpha x^{\beta}\left[\beta(r-b x)+\frac{1}{2} \beta(\beta-1) \mu^{2}\right.} \\
& \left.\quad+\int_{Y}\left\{(1+c)^{\beta}-1-\beta c\right\} v(d z)\right] \triangleq f(x) g(\beta)=0,
\end{aligned}
$$


where

$$
\begin{aligned}
g(\beta)= & \beta(r-b x)+\frac{1}{2} \beta(\beta-1) \mu^{2} \\
& +\int_{Y}\left\{(1+c)^{\beta}-1-\beta c\right\} v(d z)
\end{aligned}
$$

is well defined.

Lemma 1. $g(\beta)=0$ has two distinct real roots, the largest one, $\beta_{2}$, of which satisfies

$$
0<\beta_{2}<1 .
$$

Proof. The function $g(\beta)$ is decomposed into the sum of two functions

$$
\begin{aligned}
& g_{1}(\beta)=\beta\left(\frac{1}{2}(\beta-1) \mu^{2}+r-b x\right), \quad \forall x<K, \\
& g_{2}(\beta)=\int_{Y}\left\{(1+c)^{\beta}-1-\beta c\right\} v(d z) .
\end{aligned}
$$

Since the former $g_{1}$ is a mixture of convex exponential function $(1+c)^{\beta}$ ( $c$ is bounded), we assume that $g(\beta)$ is strictly convex function. Furthermore, we have

$$
\begin{aligned}
& g(0)=0, \\
& g(1)=b(K-x)>0 ;
\end{aligned}
$$

therefore, the nonlinear equation $g(\beta)=0$ has two distinct real roots $\beta_{1}, \beta_{2}$ such that $\beta_{1}=0$ and $0<\beta_{2}<1$, respectively.

We assume the following.

\section{Assumption 1}

$$
0<\beta<\beta_{2} \text {. }
$$

Assumption 2

$$
\frac{1}{2} \mu^{2} p \beta(\beta-1)+\int_{Y}\left(p(1+c)^{\beta}-p-c p \beta\right) v(d z)
$$

$$
<0 \text {. }
$$

Now, let us define a function $f^{*}: \mathbb{R}_{+} \rightarrow \mathbb{R}$ by

$$
f^{*}(x)= \begin{cases}f(x)=\alpha^{*} x^{\beta_{2}}, & 0<x<x^{*} \\ R(x)=p x^{\beta}-q, & x \geq x^{*},\end{cases}
$$

where $\alpha^{*}$ and $x^{*}>0$ are constants which are uniquely determined by the following equations $[2,18]$ :

Value matching condition:

$$
\begin{aligned}
f\left(x^{*}\right) & =R\left(x^{*}\right), \\
f^{\prime}\left(x^{*}\right) & =R^{\prime}\left(x^{*}\right) .
\end{aligned}
$$

That is,

$$
\begin{aligned}
& \alpha^{*}=\frac{q \beta}{\beta_{2}-\beta}\left(\frac{q \beta_{2}}{p\left(\beta_{2}-\beta\right)}\right)^{\beta_{2} / \beta}, \\
& x^{*}=\left(\frac{q \beta_{2}}{p\left(\beta_{2}-\beta\right)}\right)^{1 / \beta} .
\end{aligned}
$$

Lemma 2. Under Assumptions 1 and 2, the function $f^{*}$ : $\mathbb{R}_{+} \rightarrow \mathbb{R}$ satisfies the following properties (1)-(4).

(1) For any $x \in \mathbb{R}_{+}$,

$$
f^{*}(x) \geq R(x) .
$$

(2) $f^{*}(x)$ is strictly increasing in $x$.

(3) For any $x \in \mathbb{R}_{+}\left(x \neq x^{*}\right)$,

$$
\left[\mathscr{L} f^{*}\right](x) \leq 0 .
$$

(4) For any $x \in \mathbb{R}_{+}$, either ineq. (23) or (24) holds with equality.

Proof.

(1) Setting $y(x)=f(x)-R(x)=\alpha^{*} x^{\beta_{2}}-p x^{\beta}+q$ and differentiating $y(x)$ with respect to $x$, we have

$$
\begin{aligned}
y^{\prime}(x) & =f^{\prime}(x)-R^{\prime}(x) \\
& =x^{\beta-1}\left(\alpha^{*} \beta_{2} x^{\beta_{2}-\beta}-p \beta\right) \begin{cases}<0, & 0<x<x^{*} \\
\geq 0, & x \geq x^{*} ;\end{cases}
\end{aligned}
$$

hence, under Assumption 1, $\alpha^{*} \beta_{2} x^{\beta_{2}-\beta}-p \beta$ is an increasing function on $[0, \infty)$, and we obtain the conclusion with the help of the fact that $y(0)=q, y\left(x^{*}\right)=0, y(+\infty)=+\infty$.

(2) It is obvious.

(3) For $0<x<x^{*}, f^{*}(x)=f(x)$, we have

$$
\left[\mathscr{L} f^{*}\right](x)=[\mathscr{L} f](x)=0
$$

from (12).

For $x>x^{*}, f^{*}(x)=R(x)=p x^{\beta}-q$, we obtain

$$
\begin{aligned}
& {\left[\mathscr{L}_{f}\right](x)=[\mathscr{L} R](x)=x^{\beta}[p \beta(r-b x)} \\
& \quad+\frac{1}{2} \mu^{2} p \beta(\beta-1) \\
& \left.\quad+\int_{Y}\left(p(1+c)^{\beta}-p-c p \beta\right) v(d z)\right]=p \beta x^{\beta}(r \\
& \quad-b x)+x^{\beta}\left[\frac{1}{2} \mu^{2} p \beta(\beta-1)\right. \\
& \left.+\int_{Y}\left(p(1+c)^{\beta}-p-c p \beta\right) v(d z)\right] \leq 0,
\end{aligned}
$$

under Assumption 2. We finished the proof of (3).

(4) It is trivial from (1) and (3).

Now let us give the main theorem.

Theorem 3. Under Assumptions 1 and 2, the function $f^{*}$ : $\mathbb{R}_{+} \rightarrow R$ is the optimal value function; that is,

$$
v^{*}(x)=f^{*}(x), \quad x \in R_{+} .
$$

Moreover, the optimal stopping region $A\left(\subset \mathbb{R}_{+}\right)$and the optimal stopping time $\tau^{*}$ are given by the following:

$$
\begin{aligned}
A & =\left\{x \in \mathbb{R}_{+}: f^{*}(x)=R(x)\right\}=\left[x^{*},+\infty\right\}, \\
\tau^{*} & :=\inf \left\{t \in \mathbb{R}_{+}: X_{t}^{*} \in A\right\} .
\end{aligned}
$$


Proof. Using the function $f^{*}: \mathbb{R}_{+} \rightarrow \mathbb{R}$, we define a new stochastic process $\mathscr{M}=\left\{M_{t} ; t \in R_{+}\right\}$by

$$
\begin{aligned}
M_{t}= & e^{-\alpha t} f^{*}\left(X_{t}^{x}\right)-f^{*}(x)+\int_{0}^{t} e^{-\alpha s}\left[\mathscr{L} f^{*}\right]\left(X_{s}^{x}\right) d s \\
& +\widetilde{M}_{t},
\end{aligned}
$$

where

$$
\begin{aligned}
\widetilde{M_{t}}= & \int_{0}^{t} \mu X_{t}^{x} f^{* \prime}\left(X_{t}^{x}\right) d B_{t} \\
& +\int_{0}^{t} \int_{Y}\left(f^{*}\left(X_{t}^{x}+c X_{t}^{x}\right)-f^{*}\left(X_{t}^{x}\right)\right) \widetilde{N}(d t, d z)
\end{aligned}
$$

is a continuous local martingale, and by applying the Itô formula for the process $e^{-\alpha t} f^{*}\left(X_{t}^{x}\right)$, we obtain $M_{t}=0$.

Lemma 2 (4) implies

$$
e^{-\alpha t} f^{*}\left(X_{t}^{x}\right) \leq f^{*}(x)+\widetilde{M}_{t},
$$

with the help of the optimal sample theorem for martingale; we have, for any stopping time $\tau$ for the process $\left\{\widetilde{M}_{t}\right\}_{t \geq 0}$,

$$
\mathbb{E} e^{-\alpha(t \wedge \tau)} f^{*}\left(X_{t \wedge \tau}^{x}\right) \leq f^{*}(x)+\mathbb{E} \widetilde{M}_{t \wedge \tau},
$$

which can be written by

$$
\mathbb{E} e^{-\alpha(t \wedge \tau)} f^{*}\left(X_{t \wedge \tau}^{x}\right) \leq f^{*}(x),
$$

by noting the obvious fact $\mathbb{E} \widetilde{M}_{t \wedge \tau}=0$. lemma,

Taking lim inf $f_{t \rightarrow \infty}$ of both sides of (35), we have, by Fatou

$$
\mathbb{E}\left[e^{-\alpha \tau} f^{*}\left(X_{t}^{x}\right) I_{\{\tau<\infty\}}\right] \leq f^{*}(x) ;
$$

moreover, since the function $f^{*}$ has property Lemma 2 (1), it holds that

$$
\begin{aligned}
& \mathbb{E}\left[e^{-\alpha \tau} R\left(X_{t}^{x}\right) I_{\{\tau<\infty\}}\right] \leq \mathbb{E}\left[e^{-\alpha \tau} f^{*}\left(X_{t}^{x}\right) I_{\{\tau<\infty\}}\right] \\
& \quad \leq f^{*}(x) .
\end{aligned}
$$
(30)

On the other hand, for the stopping time $\tau^{*}$ defined by

$$
\mathbb{E}\left[e^{-\alpha\left(\tau^{*} \wedge t\right)} f^{*}\left(X_{\tau^{*} \wedge t}^{x}\right)\right]=f^{*}(x) .
$$

By the properties of Lemma 2 (1)-(4) of the function $f^{*}$, we assure that $A=\left[x^{*}, \infty\right)$ and by the properties of Lemma 2 (2), it holds that

$$
0<f^{*}\left(X_{\tau^{*} \wedge t}^{x}\right) \leq f^{*}\left(x^{*}\right) \text {, a.s. }
$$

Taking $\lim _{t \in \infty}$ of both sides of (38), we have, by the bounded convergence theorem of Lebesgue,

$$
\begin{aligned}
f^{*}(x) & =\mathbb{E}\left[e^{-\alpha \tau^{*}} f^{*}\left(X_{\tau^{*}}^{x}\right) I_{\left\{\tau^{*}<\infty\right\}}\right] \\
& =\mathbb{E}\left[e^{-\alpha \tau^{*}} R\left(X_{\tau^{*}}^{x}\right) I_{\left\{\tau^{*}<\infty\right\}}\right],
\end{aligned}
$$

where the second equality follows from the fact that, on the event $\left\{\tau^{*}<\infty\right\}$,

$$
f^{*}\left(X_{\tau^{*}}^{x}\right)=R\left(X_{\tau^{*}}^{x}\right) .
$$

Then we conclude that

$$
f^{*} \leq \mathbb{E}\left[e^{-\alpha \tau^{*}} R\left(X_{\tau^{*}}^{x}\right) I_{\left\{\tau^{*}<\infty\right\}}\right] \leq f^{*} ;
$$

that is,

$$
v^{*}(x)=f^{*}(x)=\mathbb{E}\left[e^{-\alpha \tau^{*}} R\left(X_{\tau^{*}}^{x}\right) I_{\left\{\tau^{*}<\infty\right\}}\right] .
$$

\section{Competing Interests}

The authors declare that they have no competing interests.

\section{Acknowledgments}

Yang Sun is supported by the NSFC Grant (no. 51406044 and no. 11401085) and Natural Science Foundation of the Education Department of Heilongjiang Province (Grant no. 12521116). Xiaohui Ai is supported by the NSFC Grant (no. 11401085) and the Fundamental Research Funds for the Central Universities (no. 2572015BB14).

\section{References}

[1] C. W. Clark, Mathematical Bioeconomics. The Optimal Management of Renewal Resources, John Wiley \& Sons, New York, NY, USA, 2nd edition, 1990.

[2] S. Dayanik and I. Karatzas, "On the optimal stopping problem for one-dimensional diffusions," Stochastic Processes and Their Applications, vol. 107, no. 2, pp. 173-212, 2003.

[3] M. Dai and Y. K. Kwok, "Characterization of optimal stopping regions of American Asian and lookback options," Mathematical Finance, vol. 16, no. 1, pp. 63-82, 2006.

[4] E. L. Presman and I. M. Sonin, "On an optimal stopping problem for random variables defined on a Markov chain," Theory of Probability \& Its Applications, vol. 54, no. 3, pp. 599-608, 2009.

[5] S. Christensen and A. Irle, "A harmonic function technique for the optimal stopping of diffusions," Stochastics, vol. 83, no. 4-6, pp. 347-363, 2011.

[6] E. M. Lungu and B. Øksendal, "Optimal harvesting from a population in a stochastic crowded environment," Mathematical Biosciences, vol. 145, no. 1, pp. 47-75, 1997.

[7] X. Sun and Y. Wang, "Stability analysis of a stochastic logistic model with nonlinear diffusion term," Applied Mathematical Modelling, vol. 32, no. 10, pp. 2067-2075, 2008.

[8] M. Liu and K. Wang, "Asymptotic properties and simulations of a stochastic logistic model under regime switching," Mathematical and Computer Modelling, vol. 54, no. 9-10, pp. 2139-2154, 2011.

[9] M. Liu and K. Wang, "Stationary distribution, ergodicity and extinction of a stochastic generalized logistic system," Applied Mathematics Letters, vol. 25, no. 11, pp. 1980-1985, 2012.

[10] M. Liu and K. Wang, "On a stochastic logistic equation with impulsive perturbations," Computers \& Mathematics with Applications, vol. 63, no. 5, pp. 871-886, 2012. 
[11] D. Ryan and F. B. Hanson, "Optimal harvesting of a logistic population in an environment with stochastic jumps," Journal of Mathematical Biology, vol. 24, no. 3, pp. 259-277, 1986.

[12] I.-S. Wee, "Stability for multidimensional jump-diffusion processes," Stochastic Processes and Their Applications, vol. 80, no. 2, pp. 193-209, 1999.

[13] H. Kunita, "Itô's stochastic calculus: its surprising power for applications," Stochastic Processes and Their Applications, vol. 120, no. 5, pp. 622-652, 2010.

[14] J. Bao, X. Mao, G. Yin, and C. Yuan, "Competitive LotkaVolterra population dynamics with jumps," Nonlinear Analysis. Theory, Methods \& Applications. Series A: Theory and Methods, vol. 74, no. 17, pp. 6601-6616, 2011.

[15] L. H. R. Alvarez and L. A. Shepp, "Optimal harvesting of stochastically fluctuating populations," Journal of Mathematical Biology, vol. 37, no. 2, pp. 155-177, 1998.

[16] W. Li and K. Wang, "Optimal harvesting policy for general stochastic logistic population model," Journal of Mathematical Analysis and Applications, vol. 368, no. 2, pp. 420-428, 2010.

[17] W. Li, K. Wang, and H. Su, "Optimal harvesting policy for stochastic Logistic population model," Applied Mathematics and Computation, vol. 218, no. 1, pp. 157-162, 2011.

[18] A. Dixit, The Art of Smooth Pasting, Harwood Academic Publishers, Chur, Switzerland, 1993. 


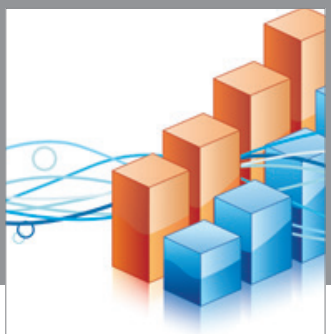

Advances in

Operations Research

vatem alat4

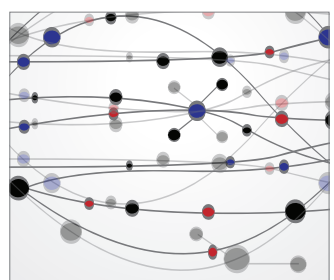

\section{The Scientific} World Journal
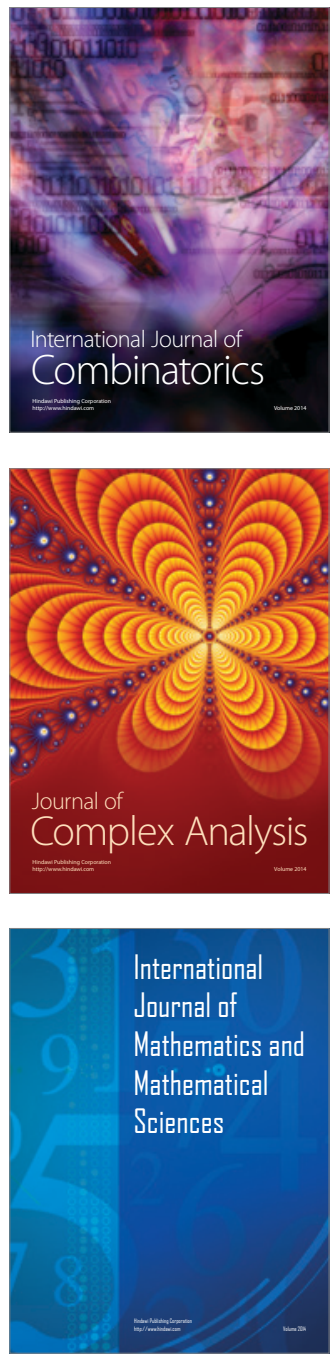
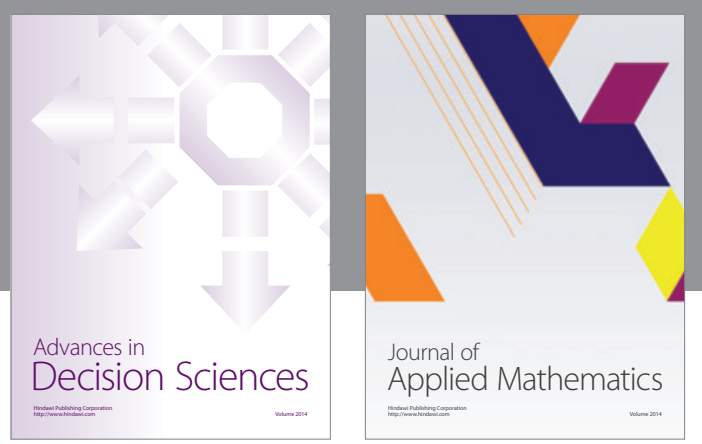

Algebra

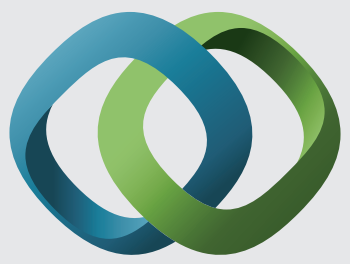

\section{Hindawi}

Submit your manuscripts at

http://www.hindawi.com
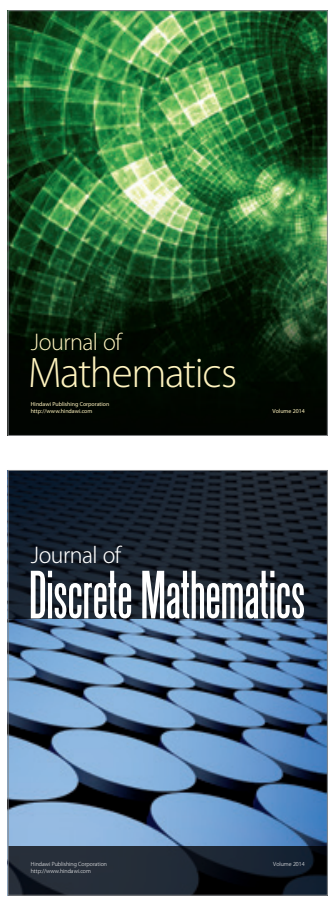

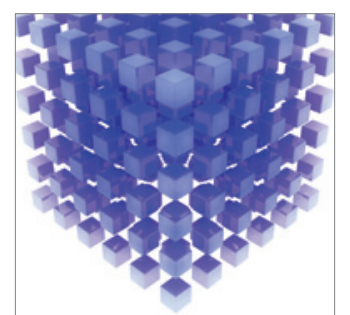

Mathematical Problems in Engineering
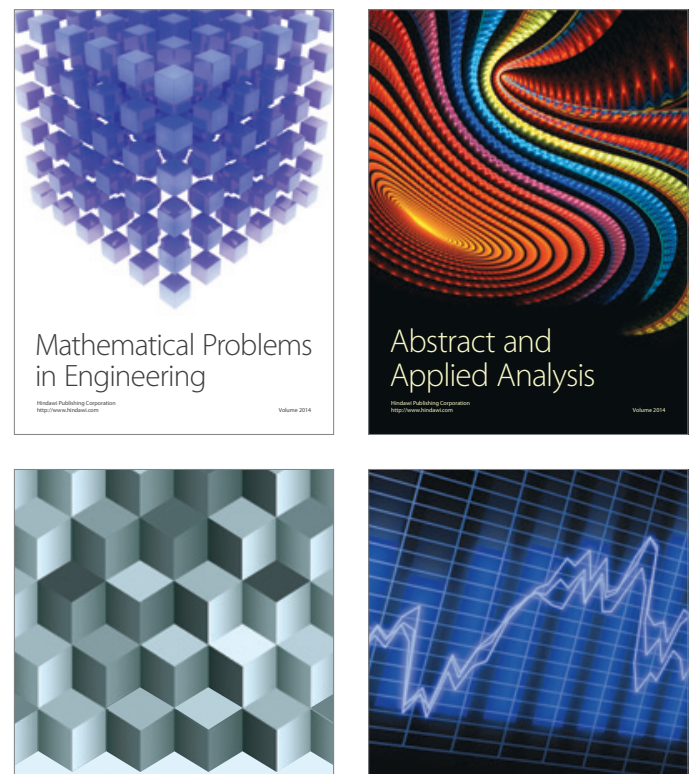

Journal of

Function Spaces

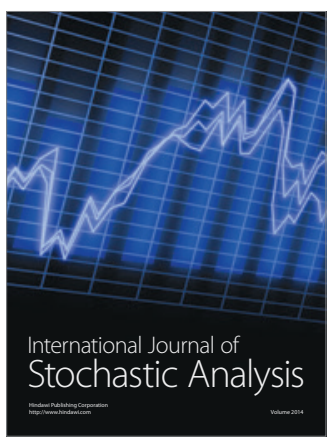

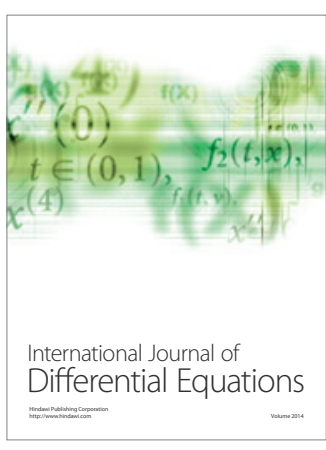
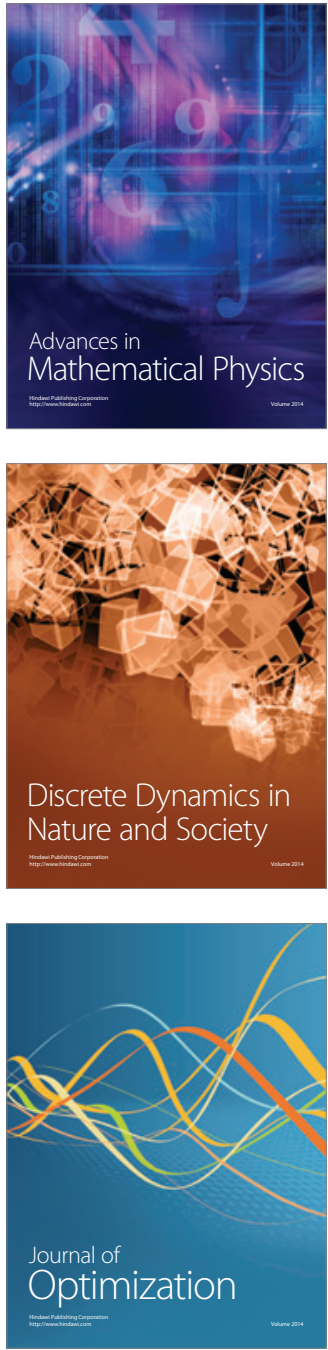